\title{
Transition between two dendritic growth mechanisms in electrodeposition
}

\author{
Graciela González, ${ }^{*}$ Michel Rosso, ${ }^{\dagger}$ and Elisabeth Chassaing ${ }^{\ddagger}$ \\ Physique de la Matière Condensée, CNRS-Ecole Polytechnique, F91128 Palaiseau Cedex, France \\ (Received 21 January 2008; revised manuscript received 9 June 2008; published 14 July 2008)
}

\begin{abstract}
We report in this paper the observation of a transition between two different dendritic growth mechanisms in the electrodeposition of a metal from a binary electrolyte. Our results, in particular concerning the dendritic growth velocities, enable us to explain this behavior in terms of models previously proposed in the literature.
\end{abstract}

DOI: 10.1103/PhysRevE.78.011601

\section{INTRODUCTION}

Electrodeposition of metals and alloys has been performed for more than a century, allowing the manufacture of a large number of metallic objects. More recently electrodeposition has emerged as a method for the synthesis of semiconductor thin films and nanostructures [1]. The flexibility of electrochemistry enables production of objects in many different states (homogeneous and uniform layers, powders, rough or porous deposits, and more recently nanostructured compounds). However, controlling the morphology of the deposit may be difficult; in particular, much effort has been devoted to preventing dendritic deposition, which may be a serious problem in battery technology [2]. Dendritic growth in electrodeposition has also been considered in the framework of out-of-equilibrium phenomena, as a model system in which several control parameters can be easily tuned [3].

In this paper, we will consider the case of electrodeposition from a binary electrolyte (an electrolyte with only one cationic and one anionic species, without supporting electrolyte). In such systems, dendritic electrodeposition has been widely investigated in the last 20 years (see [4] and references therein). Several models have been proposed to explain these phenomena: Chazalviel [5] and Elezgaray et al. [6] proposed two alternative models to explain dendritic growth in the case of deposition under high-current-density conditions, whereas Monroe and Newman [7] proposed a model valid at low current density. Indeed, as emphasized by Monroe and Newman [7], one may expect that the dendritic growth mechanism depends on the value of the applied current density, compared to the limiting current density $J^{*}$ (see below). We will present here an original situation where, due to the decrease of the distance between the deposit and the anode, the deposit growth induces a transition between the two different growth mechanisms occurring below and above $J^{*}$.

Morphological transitions are often observed in the formation of irregular electrodeposits [8-19]. For example, the

\footnotetext{
*Permanent address: INQUIMAE, Departamento de Química Inorgánica, Analítica y Química Física/Departamento de Computación, Laboratorio de Sistemas Complejos, Facultad de Ciencias Exactas y Naturales. Universidad de Buenos Aires, Ciudad Universitaria, Pabellón 2, C1428EHA Buenos Aires, Argentina.

†michel.rosso@polytechnique.fr

\$Permanent address: IRDEP, UMR 7174 EDF-CNRS-ENSCP, 6 Quai Watier, 78401 Chatou, France.
}

PACS number(s): 68.70.+w, 81.15.Pq, 82.45.Qr, 61.43.Hv

Hecker transition [8] appears as a sudden change in the color, growth velocity, and morphology of copper deposits grown from $\mathrm{CuSO}_{4}$ aqueous solutions $[8,12,15,19]$. It was shown that these changes result from the interaction of the growing deposit with a chemical front advancing from the anode toward the cathode $[12,15]$. Consequently, the transition appears on an envelope whose shape is similar to that of the anode. Another example, with alternating morphology transitions between dendritic and dense morphologies, was observed in the electrodeposition of iron from $\mathrm{FeSO}_{4}$ aqueous solutions [14]. In this case, transitions occur on each branch independently. This phenomenon was ascribed to the periodic accumulation and depletion of $\mathrm{H}^{+}$impurities in front of the growing interface.

We will consider here a slightly different situation, where the transition occurs when the current density is equal to the limiting current density $J^{*}$. In the case of a binary electrolyte with a metallic cation $M^{n+}$, if the electrodes are made of this same metal, when a current goes through the cell, cations are reduced at the cathode, and are released at the anode. On the other hand, anions do not participate in the electrode reactions. As a consequence, the ionic concentration decreases at the cathode, and increases at the anode. This effect is limited by diffusion of the anions.

According to Bard and Faulkner [20], the evolution of anionic and cationic concentrations $C_{a}$ and $C_{c}$ in our system may be described by the following set of equations:

$$
\begin{aligned}
& \frac{\partial C_{c}}{\partial t}=D_{c} \frac{\partial^{2} C_{c}}{\partial x^{2}}+\mu_{c} \frac{\partial}{\partial x}\left(C_{c} \frac{\partial V}{\partial x}\right), \\
& \frac{\partial C_{a}}{\partial t}=D_{a} \frac{\partial^{2} C_{a}}{\partial x^{2}}+\mu_{a} \frac{\partial}{\partial x}\left(C_{a} \frac{\partial V}{\partial x}\right),
\end{aligned}
$$

where $D_{a}$ and $D_{c}$ are the anionic and cationic diffusion constants, respectively, $\mu_{a}$ and $\mu_{c}$ are the anionic and cationic mobilities, respectively, and $V$ is the electrostatic potential.

Because of the above mentioned fact that the current at the electrodes is due only to cations, the boundary conditions at the electrodes may be written

$$
\begin{gathered}
-D_{c} d C_{c} / d x-\mu_{c} C_{c} d V / d x=J / z_{c} e \text { for } x=0 \text { and } l, \\
-D_{a} d C_{a} / d x+\mu_{a} C_{a} d V / d x=0 \text { for } x=0 \text { and } l,
\end{gathered}
$$

where $J$ is the current density and $e$ is the elementary charge. $l$ is the distance between the electrodes, $x=0$ is the position of the negative electrode, and $x=l$ is the position of the positive electrode. 
We now assume electroneutrality of our system, $z_{a} C_{a}$ $\approx z_{c} C_{c} \approx C$, where $z_{a}$ and $z_{c}$ are the anionic and cationic charge numbers, respectively. Then one can deduce from Eqs. (3) and (4) that the concentration gradients at the electrodes may be written [21]

$$
\left.\frac{\partial C}{\partial x}\right|_{x=0}=\left.\frac{\partial C}{\partial x}\right|_{x=l}=\frac{-J}{e D\left(1+\frac{\mu_{c}}{\mu_{a}+\mu_{c}}\right)}
$$

where $D$ is the ambipolar diffusion coefficient, given by $D$ $=\left(\mu_{a} D_{c}+\mu_{c} D_{a}\right) /\left(\mu_{c}+\mu_{a}\right)$. Now, depending on the distance $l$ between the electrodes, there are two possible regimes. At low current density, the system will eventually tend to a steady state, where the concentration roughly linearly increases from a concentration $C_{0}-\Delta C$ at the cathode to a concentration $C_{0}+\Delta C$ at the anode. $C_{0}$ is the initial concentration, and the variation $\Delta C$ is given by [21]

$$
2 \Delta C=\left.l \frac{\partial C}{\partial x}\right|_{x=0}=\frac{l . J}{e D} \frac{\mu_{a}}{\mu_{a}+\mu_{c}} .
$$

The steady state is attained after a time corresponding to the diffusion time over the distance $l$ between the electrodes. Obviously, if $C_{0}<\Delta C$, the concentration at the cathode eventually decreases to zero before the steady state is attained. In this case, which corresponds to the high-currentdensity regime, the concentration at the cathode decreases to zero at the Sand time $\tau_{s}[22]$ :

$$
\tau_{s}=\pi D\left(\frac{z_{c} C_{c 0}}{2 J}\right)^{2}\left[\left(\frac{\mu_{a}+\mu_{c}}{\mu_{a}}\right)\right]^{2},
$$

where $C_{c 0}$ is the initial cationic concentration.

The current density $J^{*}$ separating the two regimes corresponds to the limiting diffusion current observed for classical electrochemical conditions $[21,23,24]$, where the thickness of the diffusion layer would be equal to half the distance between the electrodes. From the above discussion, $J^{*}$ corresponds to the condition $C_{0}=\Delta C$; hence

$$
J^{*}=\frac{2 e D_{c} C_{0}}{l}\left(\frac{\mu_{a}+\mu_{c}}{\mu_{a}}\right) .
$$

We have assumed in the above discussion that diffusion constants and mobilities are independent of concentration: this is certainly not true in our system, but this approximation enables one to give a simple and adequate description of its behavior.

In the high-current-density regime, Chazalviel [5] attributed the onset of dendritic growth to the formation of a nonclassical space charge, related to an excess of positive charges appearing in the vicinity of the cathode when the ionic concentration at the cathode goes to zero (i.e., at the Sand time). Fleury added that, at a microscopic scale, the growth was triggered by competition between nucleation and formation of this space charge [25]. However, Elezgaray et al. [6] claimed that such a departure from electroneutrality had no physical meaning, and that this departure was not necessary to explain the destabilization of the electrode/ electrolyte interface responsible for dendritic growth.
Although the observation of electroconvective rolls [26] around the tips of the dendrites confirmed that positive charges may exist in the vicinity of the deposit, a quantitative experimental confirmation of one of the two models is still lacking. One reason is that both models predicted a similar behavior for dendritic growth, in particular that the velocity of the deposit should be equal to that of the anions in the applied electric field $E$, i.e., $v \sim-\mu_{a} E$, where $\mu_{a}$ is the mobility of the anion; this prediction has been confirmed by several authors [27-31]. At low current density $\left(J<J^{*}\right)$, Monroe and Newman proposed a different growth model [7]: this model was surface energy controlled, incorporating the effect of dendrite tip curvature into the dendrite growth kinetics. According to Monroe and Newman, dendrites accelerate across cells under all conditions (which differs from both the Chazalviel and Elezgaray et al. predictions).

In the case of a very nonuniform distribution of current densities at the electrode interface we proposed another model, which was an extension of the Chazalviel model. In particular, it predicted a constant growth velocity, equal to that predicted by Chazalviel and Elezgaray et al. This behavior has also been observed experimentally [32]. However, both the Monroe and Newman model and our model might be valid, but for different electrode/electrolyte interface conditions. We report in this paper the direct observation of a transition between the two dendritic growth mechanisms described above: the Chazalviel (or Elezgaray et al.) mechanism and a mechanism compatible with the MonroeNewman model.

\section{EXPERIMENTAL CONDITIONS}

The experiments were carried out in a pseudo-twodimensional cell [Fig. 1(a)] with $0.1 \mathrm{~mol} / \mathrm{l}$ aqueous $\mathrm{CuSO}_{4}$ solutions, at room temperature. Care was taken to obtain a well-defined cell geometry; see Ref. [33] for details. The copper electrodes were held between two glass plates enabling observation in situ of the dendritic growth [Fig. 1(b)].

The cell had a parallelepiped shape, with dimensions $L$ $\times l \times d$, where $L$ was the active length of the electrodes, $l$ the interelectrode distance, and $d$ the thickness of the cell. Practical values were $L=0.8-1.5 \mathrm{~cm}, l \approx 0.9 \mathrm{~mm}$, and $d$ $\approx 0.12 \mathrm{~mm}$. The large faces of the electrodes are coated with a hydrophobic film which limits the invasion of the electrolyte between the electrodes and the glass plates. After filling the cell with the electrolyte, we sealed it with a twocomponent resin; this allowed us to keep it under almost constant conditions for several hours [33].

Several authors have evidenced that, due to the change in concentration and to the related change in electrolyte density occurring at the electrodes, the deposition process may be accompanied by a buoyancy-driven convective motion [34-38]. This modifies concentration profiles close to the electrodes [34], and has a destabilizing effect on the deposition process [37]. In order to avoid this effect, we used a technique that is classically used in this kind of experiment [34-37,39]; it consists in setting the cell in a vertical position, with the cathode on top. In these conditions, because the light fluid is on top and the heavy one is at the bottom of 


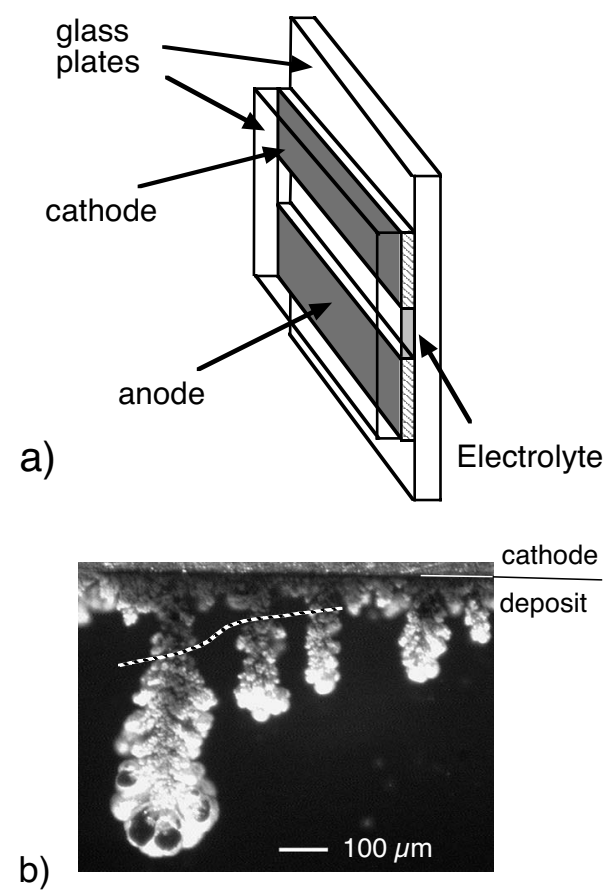

FIG. 1. (a) Experimental cell. (b) Photograph of a few dendrites: we see in this picture that the dendrite morphology changes from powderlike at the beginning of the growth, to compact afterwards; the white dotted line shows the place where this change occurs. The solid white line is $100 \mu \mathrm{m}$ long; this is also approximately the thickness of the cell. Hence, at this scale, the deposit appears as three dimensional.

the cell, buoyancy is almost suppressed. Compared to other methods, this method appeared to offer the best compromise for reducing buoyancy effects. In particular, Marshall et al. [39] showed that this configuration enables a stable growth regime to be obtained.

Of course this configuration does not prevent the occurrence of the electroconvective motion mentioned above; however, this motion is not expected to appear before the onset of irregular growth, and hence it does not perturb the initial stages of deposition. Also, electroconvection originates from the electric fields appearing at the tips of the deposit [26]; thus we believe that this motion is related basically to at least one possible mechanism of dendritic growth. While suppression or limitation of buoyancy-driven convection aims at simplifying the system under study, suppression of electroconvection might result in changing its physical nature.

In situ visualization of the cell enabled us to follow the evolution of the dendrites [Fig. 1(b)], and, simultaneously, to measure concentration maps; for this we made use of the absorption properties of $\mathrm{Cu}^{2+}$ ions [33].

\section{EXPERIMENTAL RESULTS}

We performed experiments in galvanostatic conditions, for constant current densities ranging from $J^{*} / 2$ to $2.5 J^{*}$, i.e., from $1.8 \times 10^{-3}$ to $8.5 \times 10^{-3} \mathrm{~A} \mathrm{~cm}^{-2}$. At low current densities $\left(J<J^{*}\right)$, we observed that the concentration gradients

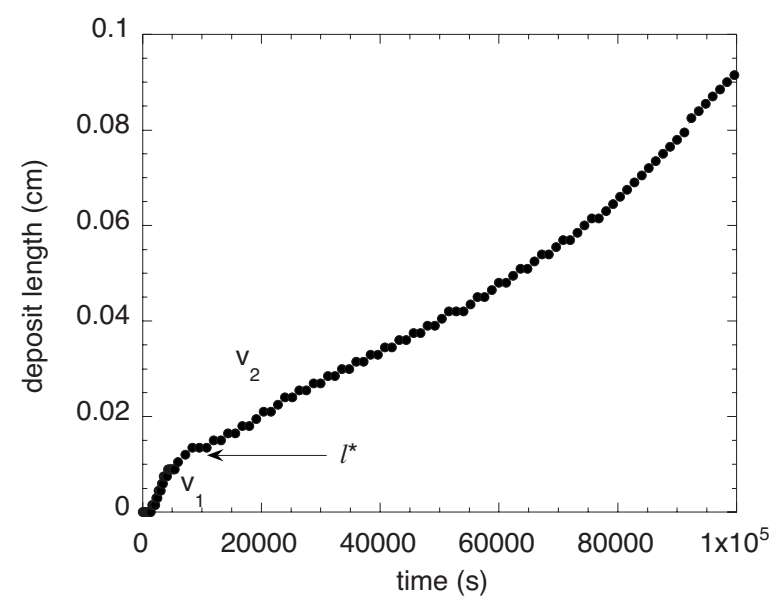

a)

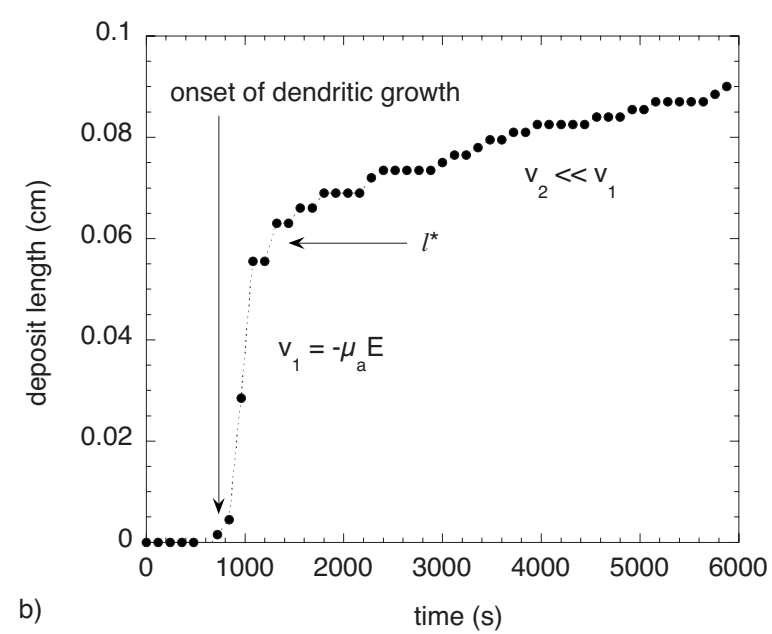

FIG. 2. Variation of dendrite growth velocity with time. The growth velocity changes from $v_{1}$, close to the Chazalviel prediction, to a much smaller value $v_{2}$. This change occurs at the same time as the dendrite structure changes [see Fig. 1(b)]. (a) The applied current $I$ is slightly above $I^{*}\left(I=1.2 \times I^{*}\right)$; (b) $I=2.5 \times I^{*}$.

were not constant throughout the cell [40]. Hence the current density was not uniform in the cell, possibly due to nonuniformity of the electrode surface. However, at high current densities, the concentration maps appeared to be much more uniform. We attribute this behavior to a more efficient deoxidation of the electrode surface at higher current densities. In any case, for a given cell, we will report in the following the maximum values of the parameters we measure in the cell, the local current density, deposit length, and velocity.

At high current densities, the early stages of deposition were similar to those reported in previous studies. We first observed an induction period, during which a variation of the concentration appeared in the cell, with the concentration decreasing at the cathode and increasing at the anode. In this period, the deposit growth was very slow, we could not observe any dendrite, and the cell potential was almost constant. When the concentration decreased to zero at the cathode, at a time close to Sand time, the cell voltage increased rapidly, then stabilized: at this moment dendrites started growing. The observed behavior was compatible with the 


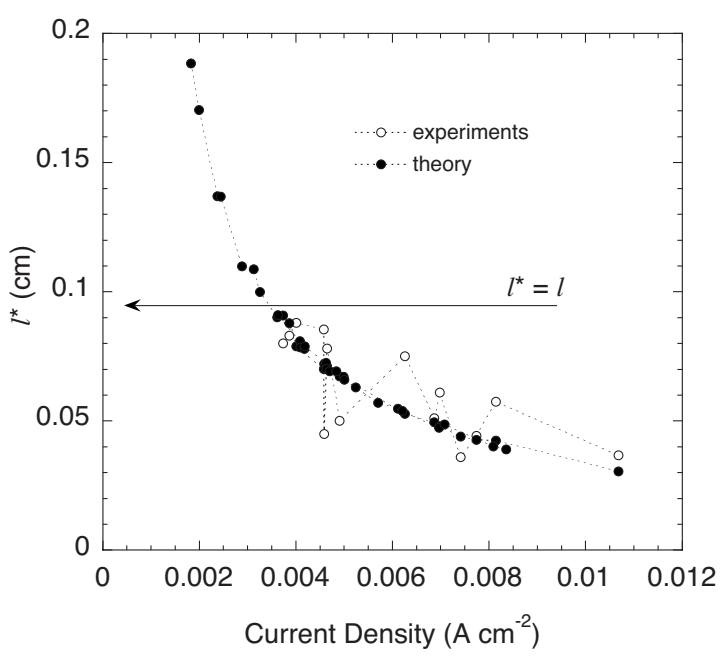

FIG. 3. Variation of the length $l^{*}$ at which we observe the dendrite morphological change shown in Fig. 1(b), with applied current density (open circles). Closed circles show the theoretical prediction [from Eq. (6)].

Chazalviel and Elezgaray et al. models; the dendrite velocity $v_{1}$ was close to the velocity of the anions, i.e., $v_{1} \sim-\mu_{a} E$.

Due to dendritic growth, the distance between the deposit and the anode rapidly decreased; consequently, $J^{*}$ increased [Eq. (3)]. When working at a constant current, the distance $l^{\prime}$ between the deposit front and the anode eventually decreased to a value $l^{*}$, at which the corresponding value for $J^{*}$ was equal to the imposed current density. We then observed that the deposit growth velocity suddenly decreased to a value $v_{2} \ll v_{1}$ (Fig. 2). We also observed a clear difference in the dendrite morphology, which was powderlike in the $v_{1}$ regime and compact in the $v_{2}$ regime [Fig. 1(b)].

The dependence of the distance $l^{*}$ on applied current density is shown in Fig. 3. Theoretical values are shown for comparison. The fluctuations observed on our experimental values are due to uncertainties on our measurements; in particular, the value of $l^{*}$ at which the transition is observed is not precisely determined (see Fig. 2).

We attribute the changes in growth velocity and dendrite structure to a transition from the high-density regime (Chazalviel or Elezgaray et al. model) to a low-currentdensity regime compatible with the Monroe-Newman model [7]). In order to further support this hypothesis, we performed experiments at current densities much lower than $J^{*}$. In this case, we observed that dendritic growth was very far from that predicted by Elezgaray et al. or Chazalviel: the variation of the cell voltage was markedly different, with no abrupt increase of the cell voltage [40]. We observed dendritic growth, but the growth velocity was not constant, increasing with the dendrite length. It was always much smaller than that expected for the anions (Fig. 4). In general, it was compatible with that observed in the $v_{2}$ regime at high current density. Also, the time at which dendrites appeared was much larger than expected from an extrapolation of the

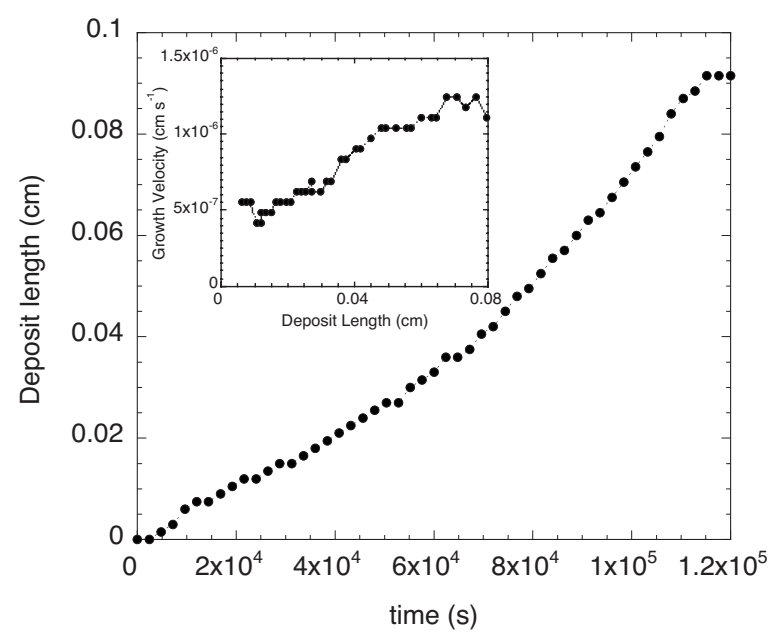

FIG. 4. Plot of the deposit length as a function of time at low applied current $I=0.86 \times I^{*}$ : this variation is in qualitative agreement with Monroe's prediction [7], as suggested from the inset, where we observe a linear dependence of the growth velocity with the deposit length.

Sand time. In general we observed only a few dendrites (possibly one), which appeared to be compact. A plot of the dendrite velocity as a function of the dendrite length is shown in the inset of Fig. 4; an almost linear increase is observed, in qualitative agreement with Monroe-Newman prediction [7].

\section{CONCLUSION}

Two dendritic growth modes were observed in metal electrodeposition from a binary electrolyte. If the applied current is larger than the limiting current, these modes can be observed consecutively in a single experiment. In this case, the transition appears at the distance from the negative electrode where the limiting current becomes equal to the applied current. At the early stage of the experiment, in agreement with earlier experiments, our results may be explained in the framework of the models proposed by Chazalviel [5] or Elezgaray et al. [4]. The second behavior, as well as that observed in experiments performed at low current density, agrees well with a model recently proposed by Monroe and Newman [7]. In the low-current-density regime, dendrites appear much later and grow much more slowly than in the high-current-density regime: hence, if one wishes to avoid dendritic growth, the former regime may be considered as more favorable than the latter.

\section{ACKNOWLEDGMENTS}

G.G. acknowledges financial support from a CNRS grant for a three-month visit at Ecole Polytechnique. We wish to thank Dr. Thierry Gacoin for his help in preparing the electrolytic cell. 
[1] D. Lincot, Thin Solid Films 487, 40 (2005).

[2] J. M. Tarascon and M. Armand, Nature (London) 414, 359 (2001).

[3] T. Vicsek, Fractal Growth Phenomena, 2nd ed. (World Scientific, Singapore, 1992).

[4] C. Léger, J. Elezgaray, and F. Argoul, J. Electroanal. Chem. 486, 204 (2000).

[5] J.-N. Chazalviel, Phys. Rev. A 42, 7355 (1990).

[6] J. Elezgaray, C. Léger, and F. Argoul, J. Electrochem. Soc. 145, 2016 (1998).

[7] C. Monroe and J. Newman, J. Electrochem. Soc. 150, A1377 (2003).

[8] N. Hecker, D. G. Grier, and L. M. Sander, in Fractal Aspects of Materials, edited by R. B. Laibowitz, B. B. Mandelbrot, and D. E. Passoja (Materials Research Society, University Park, PA, 1985).

[9] P. Garik, D. Barkey, E. Ben-Jacob, E. Bochner, N. Broxholm, B. Miller, B. Orr, and R. Zamir, Phys. Rev. Lett. 62, 2703 (1989).

[10] J. R. Melrose, D. B. Hibbert, and R. C. Ball, Phys. Rev. Lett. 65, 3009 (1990).

[11] M. A. Guzman, R. D. Freimuth, P. U. Pendse, M. C. Veinott, and L. Lam, in Nonlinear Structures in Physical Chemistry, edited by L. Lam and H. C. Morris (Springer, New York, 1990), p. 32.

[12] V. Fleury, M. Rosso, and J.-N. Chazalviel, Phys. Rev. A 43, 6908 (1991).

[13] P. P. Trigueros, J. Claret, F. Mas, and F. Sagués, J. Electroanal. Chem. Interfacial Electrochem. 312, 219 (1991).

[14] M. Wang and N. B. Ming, Phys. Rev. Lett. 71, 113 (1993).

[15] A. Kuhn and F. Argoul, Phys. Rev. E 49, 4298 (1994).

[16] M.-Q. Lòpez-Salvans, F. Sagués, J. Claret, and J. Bassas, J. Electroanal. Chem. 421, 205 (1997).

[17] M.-Q. Lòpez-Salvans, F. Sagués, J. Claret, and J. Bassas, Phys. Rev. E 56, 6869 (1997).

[18] K. Q. Zhang, M. Wang, S. Zhong, G. X. Chen, and N. B. Ming, Phys. Rev. E 61, 5512 (2000).

[19] G. González, G. Marshall, F. V. Molina, S. Dengra, and M. Rosso, J. Electrochem. Soc. 148, C479 (2001).
[20] A. J. Bard and L. R. Faulkner, Electrochemical Methods. Fundamentals and Applications (Wiley, New York, 1980).

[21] C. Brissot, M. Rosso, J.-N. Chazalviel, and S. Lascaud, J. Power Sources 81-82, 925 (1999).

[22] H. J. S. Sand, Philos. Mag. 1, 45 (1901).

[23] S. Atlung, K. West, and T. Jacobsen, J. Electrochem. Soc. 126, 1311 (1979).

[24] P. G. Bruce and C. A. Vincent, J. Electroanal. Chem. 225, 1 (1987).

[25] V. Fleury, Nature (London) 390, 145 (1997).

[26] V. Fleury, J.-N. Chazalviel, and M. Rosso, Phys. Rev. Lett. 68, 2492 (1992).

[27] V. Fleury, J.-N. Chazalviel, M. Rosso, and B. Sapoval, J. Electroanal. Chem. Interfacial Electrochem. 290, 249 (1990).

[28] J. R. Melrose, D. B. Hibbert, and R. C. Ball, Phys. Rev. Lett. 65, 3009 (1990).

[29] P. P. Trigueros, F. Sagués, and J. Claret, Phys. Rev. E 49, 4328 (1994).

[30] K. Linehan and J. R. de Bruyn, Can. J. Phys. 73, 177 (1995).

[31] C. Léger, J. Elezgaray, and F. Argoul, Phys. Rev. E 58, 7700 (1998).

[32] M. Rosso, T. Gobron, C. Brissot, J.-N. Chazalviel, and S. Lascaud, J. Power Sources 97-98, 804 (2001).

[33] M. Rosso, E. Chassaing, J.-N. Chazalviel, and T. Gobron, Electrochim. Acta 47, 1267 (2002).

[34] M. Rosso, J.-N. Chazalviel, V. Fleury, and E. Chassaing, Electrochim. Acta 39, 507 (1994).

[35] D. P. Barkey, D. Watt, Z. Liu, and S. Raber, J. Electrochem. Soc. 141, 1206 (1994).

[36] J. Huth, H. Swinney, W. McCormick, A. Kuhn, and F. Argoul, Phys. Rev. E 51, 3444 (1995).

[37] G. Marshall, E. Perone, P. Tarela, and P. Mocskos, Chaos, Solitons Fractals 6, 315 (1995).

[38] G. Marshall, P. Mocskos, H. L. Swinney, and J. M. Huth, Phys. Rev. E 59, 2157 (1999).

[39] G. Marshall, E. Mocskos, G. González, S. Dengra, F. V. Molina, and C. Iemmi, Electrochim. Acta 51, 3058 (2006).

[40] G. González, E. Chassaing and M. Rosso (unpublished). 